Session 1135

\title{
A Study of Mainstream Features of CRM System And Evaluation Criteria
}

\author{
Melissa C. Lin \\ Information Technology Specialist \\ U.S. Department of Agriculture
}

\begin{abstract}
This paper will discuss an overview of Customer Relationship Management (CRM) systems, mainstream CRM features and functionalities, CRM evaluation criteria and vendors selection. It will also include selecting a piece of enterprise application software, resolving a conflict of vendor solution and client's requirements. The paper provides the study of the CRM features and evaluation criteria is based on a small to medium manufacturing company. A comparison table was prepared for helping the company to select an appropriate vendor.
\end{abstract}

\section{Introduction}

As companies face increasing competition, wider customer choice, and the challenges of doing e-Business in the $21^{\text {st }}$ century, many have chosen to implement multi-year CRM solutions in response to their strategic imperative, and to improve the sales and marketing effectiveness/efficiency. A Customer Relationship Management (CRM) system is a software system designed to empower a company to maximize profits by reducing costs and increasing revenue; to increase competitive advantage by streamlining operations; and to achieve business goals. Most companies have been collecting information about their customers and trying to use this information to better understand and predict what customers might want next. Therefore, CRM is both information-based and technologybased, and is about building customer loyalty by putting customers at the center of what a company does. CRM also applies to how customers experience a company, not just how a company looks at its customers.

Many companies use CRM both as strategy and as software solutions to increase revenue through effective sales, marketing, and customer service by accessing customer information quickly and accurately. Using a set of customized software tools built around a CRM suite, the company can respond to its current business conditions and access 
information they need with flexible analysis in minutes. CRM systems also assist employees in analyzing the collected data about sales, marketing and so forth to provide accurate and useful business information such as current sales trends, customer behavior, etc., for senior executives. CRM packages give employees more power and control on when and how they want to do business. An Internet-based CRM enables the possibility of globalization and thus opens up the entire world market place. With additional customized software and tools that make product and customer feedbacks available for Research \& Engineering, a CRM may also assist the continuing quality improvement (CQI) process and the development of new products.

This paper is divided into the following sections: Section II introduces major components of CRM; Section III depicts mainstream features of CRM; Section IV defines CRM evaluation criteria and vendors selection; finally a conclusion is given as Section V.

\section{Major Components of CRM Systems}

A successfully implemented CRM system allows seamless integration with the existing software and systems, increases productivity in time pressured situations, and supports business performance, scalability, and reliability. Through the available built-in software functionalities, a CRM system can help a company understand key customer behaviors, plan effective marketing campaigns/strategies, and increase the business growth rate.

Mainstream vendors recognize that information technology and business functions including marketing, sales, customer services and support must work together to build a successful CRM that can support a typical Customer-Life Cycle in daily business operations. A CRM hierarchy is in shown Figure 1.

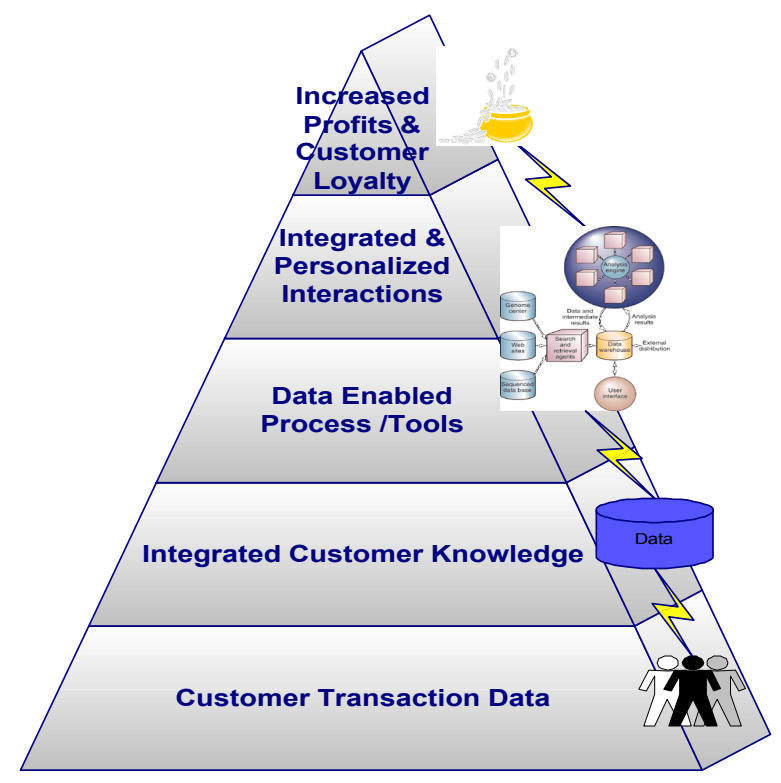

Figure 1: CRM Hierarchy

Proceedings of the 2003 American Society for Engineering Education Annual Conference \& Exposition Copyright 2003, American Society for Engineering Education 
A study from available CRM vendors indicates that the following four software modules are available:

- Sales Automation Module - This module is designed to automate sales related tasks such as sales-customer interaction, contact scheduling, sales campaign and promotion activities, sales lead tracking, sales trends and forecasting, sales knowledge exchanging, inventory control and monitoring, reducing sales cycles, etc.

- Marketing Automation Module - This module is designed to automate marketing related tasks such as marketing analysis and planning, marketing campaign activities and location, products promotion and scheduling, pricing compatibility and competition, marketing trends and forecasting, marketing knowledge exchanging, etc.

- Customer Service \& Support Module - This module is designed to document and manage customer information and activities thereby building strong and long lasting customer relationships, including unmet customers, new customers, existing customers, and valuable customers.

- Reporting and Analysis Tools - These tools are a set of software and technology to enable cross-channel, complete view of sales, marketing, and customer service information that stored on company's databases for analytic reporting and analysis. A CRM automated system evolves and interacts with various commonly used enterprise applications such an Enterprise Resource Planning (ERP) system, a Web server, database systems, and a legacy system as depicted in Figure 2. Referring to this EnterpriseApplications Integration (EAI) architecture, it is helpful to "think big but act small" while a company implements its new CRM automation system.

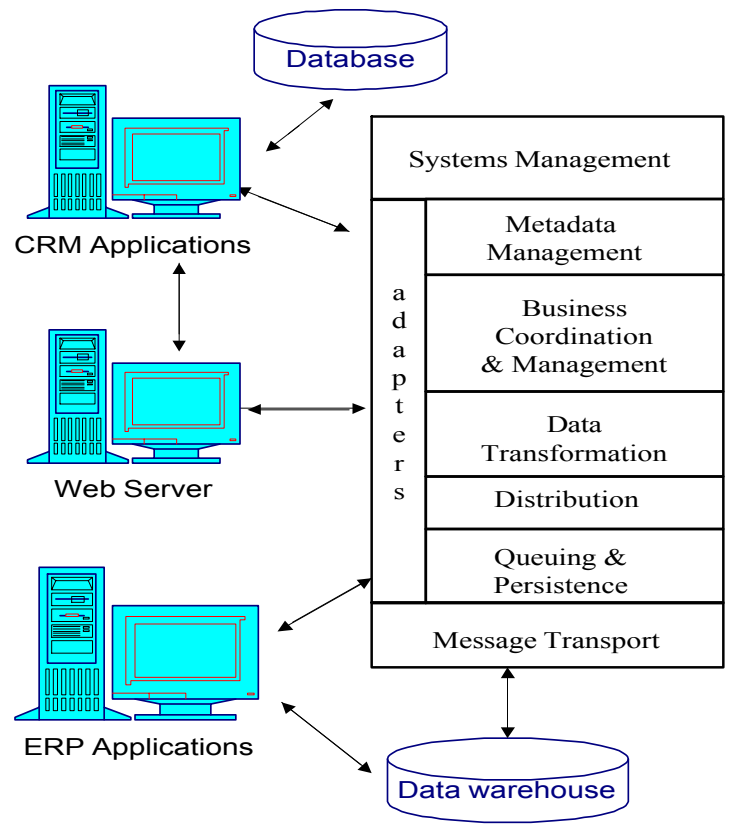

Figure 2: CRM and Enterprise Applications Integration 


\section{Mainstream Features of Customer Relationship Management Systems}

A CRM system is a Web-enabled database application that provides rapid and accurate insight information, optimizes the interaction with customers, increases the productivity and efficiency, reduces the cost, integrates dissimilar platforms, and shares data with staff and customers.

After a detailed study of mainstream CRM features, a generalized CRM architecture as shown in Figure 3 is defined. This CRM architecture should serve as a reference for evaluating and deploying a desired CRM system. On the front-end, the Communication CRM supports various mechanisms of customer communications; the Operation CRM is tailored for Sales, Marketing, and Customer Services, with extension to serve Research \& Engineering; the back-end Analytic CRM supports all the needs in Analysis and Reporting.

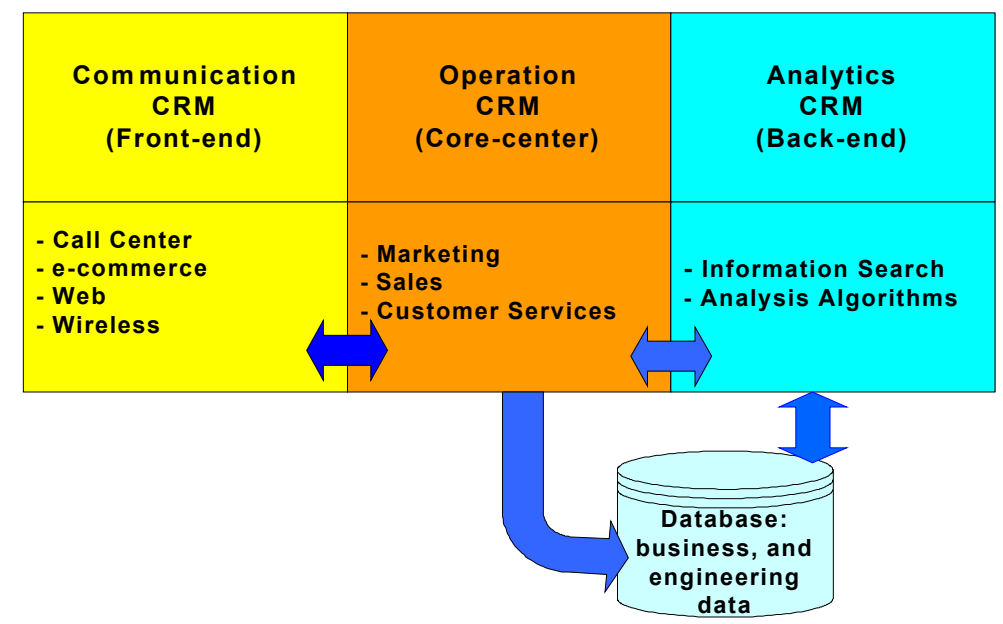

Figure 3: Generalized CRM Architecture

A CRM system is an integrated sales, service, and marketing strategy for capturing, organizing, and leveraging customer information. A comprehensive CRM strategy should ensure that information is gathered from all customer interactions with the company. The information collected on a customer should include such items as sales contact history, sales orders (including order history and current order status), products purchased, product pricing, account affiliations, contracts and compliance, entitlements and warranties, service history (including service order details), and responses to marketing campaigns. An effective CRM helps a company to increase customer value by improving customer satisfaction and strengthening customer loyalty.

To create an effective CRM strategy, the mainstream features must encompass and integrate all company activities that involve interaction with customers, including marketing, sales, customer service, and field service. It should ensure that no matter where, when, or how the customer comes in contact with the company, the response is personalized and consistent and demonstrates that the company knows and values the 
customer. Achieving this goal, the following mainstream features are expected to be available in a desired CRM application to support Sales Automation, Marketing Management, Customer Services and Supports, Research and Engineering Automation, Data Analyzing and Report Generations, and Data Mining/Data Warehouse.

- Call Management

- Customers Management

- Services Issues Management

- Knowledge Exchange Management

- Managing Promotion Activities

- Sales Force Activities

- Marketing Campaign Management

- Sale Lead Tracking

- Database Storage - Word searching and Report Generation

- Extending support for outside the office

\section{Marketing Automation Suite}

A Marketing Automation software system is designed to focus on analyzing and automating marketing activities and process, including customer interaction, product implementation, pricing adjustment, promotion designing, and location selection. Mainstream CRM vendors offer Marketing Automation with the following key features:

* Marketing Campaigns Management

* Contact Management

* Marketing Analysis and Forecasting

As highlighted in Figure 4 shows a cross-management of Marketing Automation functionalities. Marketing Automation software should include data analysis for planning the campaign, defining the targeted customers, determining the channel of location and communication, and launch promotion.

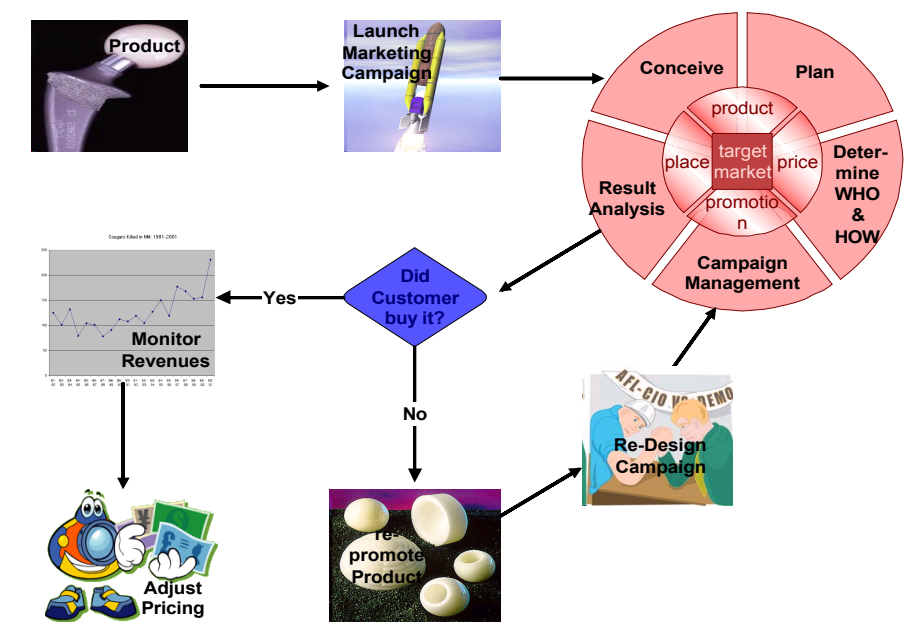

Figure 4: Functionalities of Marketing Automation 
Marketing Automation will not only include the traditional focus on branding and messaging strategies and broadcast communications, but also the new marketing skills and knowledge to ensure consistent, integrated communications and experiences for supporting the company's brand image and the customers. The new roles of marketing automation are responsible for decision on the designing of marketing process and management activities as the following:

- $\quad$ Selecting Target Market - This feature provides the analysis of what customers need, how to design a product category designs, and what is the current competition in the industry today

- Identify Market Segment

- Selecting Target Customer

- Product (definition of the "whole product")

- Price (levels and strategies)

- Promotion (Communication)

- Place (Sales/Distribution methods)

\section{Sales Automation Suite}

A Sales Automation software system is designed to automate sales related tasks such as sales activities and processes, customer interaction, scheduling and tracking, inventory control and monitoring, sales trends and forecasting, and sales lead management. It can help streamline the sales process; target the right customers; create a competitive sales climate; and assist in effective time management. It also reduces the administrative load on the sales force in accessing information of products and customers in real time, and allows sales representative to keep current on company and customers' information. Mainstream CRM vendors offer the following key features in their Sales Automation software suites:

* Personalization

* Sales Distribution Channels to support the customers and internal management

* Instant Sales Information available through accessible databases

* Sales Activity and Time Management

* Sales Account, Contact, and Opportunity Management

* Forecast Sale Trends and Revenues Accurately

* Analyze and report and Analyze of Sales Opportunities

As highlighted in Figure 5, integration of technology and Sales Automation software functionalities is an end-to-end process. Sales Automation software should have attributes such as menu-driven, user friendly, and simplicity in order to provide customer centered services, which can be accessed anywhere, anytime, through a Web browser. The deployment of such a customer-centric e-Business would also improve the chances of sales and deliver a good return on the investment. 


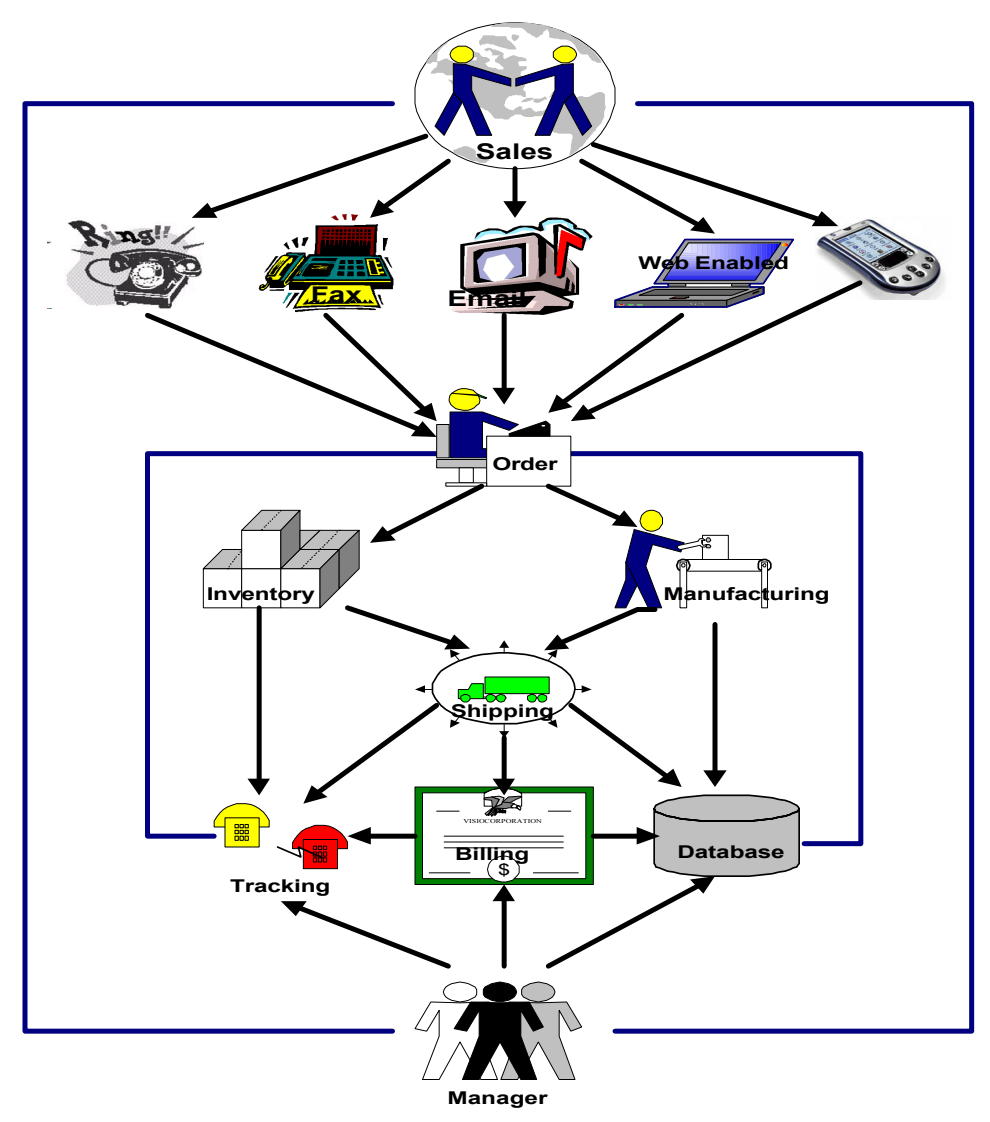

Figure 5: Functionality of Sales Automation

Due to the array of technological resources available to the contemporary sales representatives --Web sites, laptops, software, printers, modems, fax-copies, emails, cellular phones, and pagers --sales representatives are given more time for personal interaction with customers. Sales representatives serve as the company's personal links to customers. CRM software must help the company manage relationships with distributors, hospitals, clinics, and physicians - both operationally and strategically. A Sales Automation system will consist of a set of new technology-enabled programs that help both sales representatives and management increase their productivity and efficiency in performing the following sale force activities:

- Supervise sales representatives

- Visit/contact customers

- Manage contacts and relationships

- Access and analyze products

- Product presentation and demonstration

- Price negotiation

- Setup and Managing sale leads and customer information

- Evaluate competitors' business strategies

- Identify target customers for sales campaigns

- Follow-up, maintenance, and resolve problems 


\section{Customer Service \& Support Software Suite}

A Customer Service and Support Automation system is designed to automate contact centers. Contact Centers have evolved quickly from a group of local employees answering phones during business hours to a dedicated organization providing on-demand global support and sales through several different channels. Mainstream CRM vendors offer the following key features in the Customer Service and Support Automation software suites:

* Call Routing

* Contact Center Sales Support.

* Web-based Self-Service

* Customer Satisfaction Measurement

* Call- Scripting

* Workforce Management

* A Checklist of Customer Service and Support for Success

As highlighted in Figure 6, it is desirable to add customer intelligence capabilities to Customer Service and Support Automation functionalities.

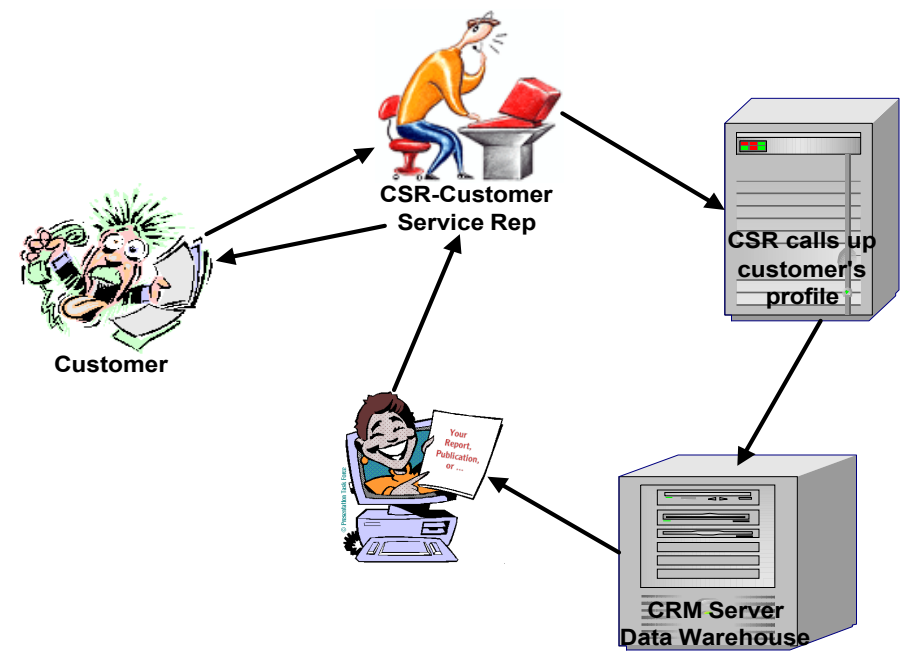

Figure 6: Functionalities of Customer Service/Support Automation

Depending on business functions, roles, and responsibilities, CRM Customer Services \& Support software suites offered by mainstream CRM vendors may include one or more of the following functions and supports:

- Company vision, mission, values, news

- E-mail management

- Wireless messaging contacts

- Computerized telephony services

- Web-based services

- Web-based customer and product information, easily accessed by employees

- Emergency announcements 


\section{Report and Data Analysis Software Suite}

An effective CRM system provides the data analysis and reports that gives instant insight into important customer trends and patterns. The information sharing and collaboration enabled by effective CRM benefit all functional groups. Therefore, the data structure employed by a CRM solution must accommodate the complexity of the product distribution chain, encompassing customer profiles, contracts and pricing, product configurations, complaints, assessments, sales forecasts, and incentive compensation. It also should have an extensive government regulation, and define the regulatory framework for manufacturers using computerized systems as repositories of product quality data, and safety reports. By selecting and deploying a comprehensive and integrated CRM software solution that meets the unique requirements, a company can ensure the effective implementation of its CRM strategy. As a result, the company can build far stronger customer relationships for improved performance and increased profitability.

These key features of report generation and analysis data tools will help employees and staffs to manage cross-channel information, complete view of sales, marketing, and customer service information stored in company's databases for reporting and analysis. The mainstream features of report and data analysis software will help measure the performance of the following business activities:

- Marketing insight

- Sales Report and Data Analysis

- Customer Service \& Support

- Profitability

\section{CRM Evaluation Criteria and Vendor Selection}

The following example of CRM evaluation criteria and vendor selection is based on a small to medium size manufacturing company. This CRM solution is designed specifically to accommodate the distinctive characteristics of a specific technology industry-including complex distribution channels with multi-tiered supply-chain relationships, complicated contracts and pricing structures, and stringent government regulation. The company's CRM strategy focuses on streamlining sales and marketing operations, reducing costs, increasing return on investment, and achieving business goals. The desired CRM will gain customer loyalty through quality services and products, effective communication, and timely solutions to address customer problems, wants and needs. The roles of the company CRM are identified as the following:

- Sales Automation

- Marketing Automation

- Customer Service \& Support Automation

- Research \& Engineering Automation

- Data Analysis and Reports

- Data warehouse

Proceedings of the 2003 American Society for Engineering Education Annual Conference \& Exposition Copyright 2003, American Society for Engineering Education 
Figure 7 shows the proposed a company CRM system:

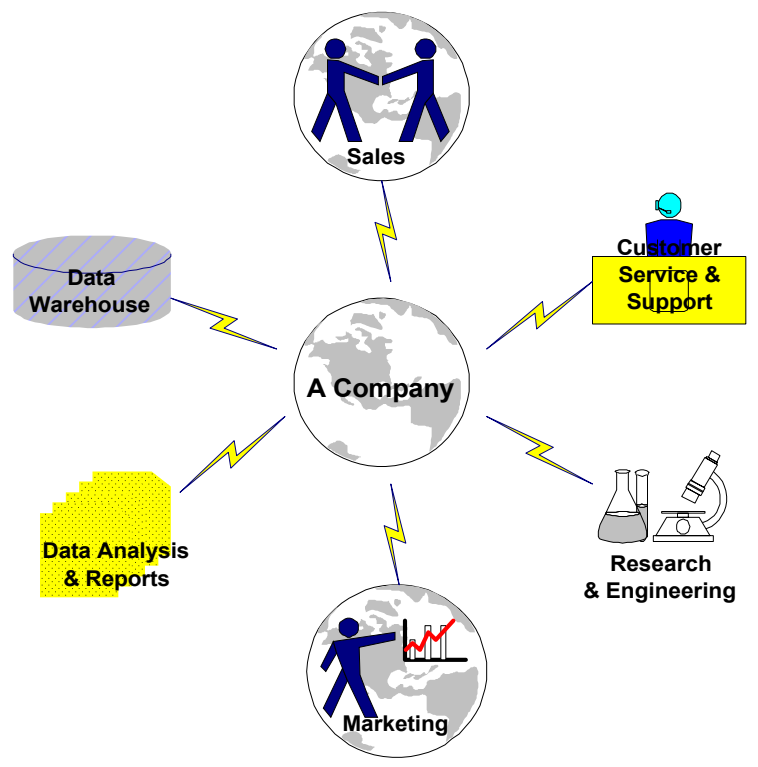

Figure 7: A Company CRM

In addition to the desired features and mission of a desired CRM system, we assume a rough-draft of minimum IT technology requirements are also given below:

1) Compatibility with NT / Win2000 OS

2) Web capable

3) Open Systems - ODBC compatibility

4) A Progress based is desired (for backward compatibility) but not required

5) Main stream DB technology - none proprietary infrastructure technology

6) Client Server is not required, but the web access requirement is absolutely needed

7) Must have PDO (Palm device compatibility)

8) License Costs

The application supports both Web Clients or Client/Server based application, and can be run on a single notebook PC or on a corporate network consisting of Windows ${ }^{\mathrm{TM}}$ clients and Windows NT or UNIX operating systems. It should have the following key features:

$>$ Windows $^{\mathrm{TM}}$ Explorer style user interface

$>$ All information is accessed from one central screen

$>$ Multi-company and multi-user system

$>$ Workgroup environment including multi-user diaries and task lists

$>$ Built in management reporting and analysis tools

$>$ Facility for importing data from other sources

$>$ Interacts with Microsoft Word and Excel

$>$ Comprehensive links to Microsoft Outlook/Exchange Server

$>$ Interacts with Symantec WinFax Phonebook, Microsoft Exchange Address Book, E-Mail client, Microsoft Outlook, Palm Pilot 
Evaluation criteria A Comparison of Mainstream CRM functionalities:

\begin{tabular}{|r|l|c|c|c|}
\hline & \multicolumn{1}{|c|}{ Features/Evaluation Criteria } & ABC co & MNO co & XYZ co \\
\hline 1 & Scheduling & yes & yes & yes \\
\hline 2 & Contact Tracking & yes & yes & yes \\
\hline 3 & lustomer Management & yes & yes & yes \\
\hline 4 & Email Management & yes & yes & yes \\
\hline 5 & Issue and Request Tracking & yes & yes & yes \\
\hline 6 & Knowledge Management (Internal+ External) & yes & yes & yes \\
\hline 7 & Marketing Campaign Management & yes & yes & yes \\
\hline 8 & Order Management & yes & yes & yes \\
\hline 9 & Sales Lead Tracking & yes & yes & yes \\
\hline 10 & Trend Analysis & yes & yes & yes \\
\hline 11 & Data Storage Interface & yes & yes & yes \\
\hline 12 & Report Generation & yes & yes & yes \\
\hline 13 & Interface with Word, Excel, Outlook & yes & N/A & yes \\
\hline 14 & Compatibility with NT/Win2000 OS & yes & yes & yes \\
\hline 15 & Web Capable - Integration & N/A & yes & yes \\
\hline 16 & Palm Device Compatibility & yes & yes & yes \\
\hline 17 & ODBC Compatibility & yes & N/A & N/A \\
\hline 18 & Progress-based & & & \\
\hline
\end{tabular}

Note that $\mathrm{ABC}, \mathrm{MNO}$, and $\mathrm{XYZ}$ are pseudo companies and are used only for illustration purposes. The comparison table with features extracted from the given requirements gives the CRM evaluators a set of key information for screen vendors for valuations.

\section{Conclusion}

This paper gave an overview of CRM systems, discussed mainstream CRM features and functionalities. Selecting a piece of enterprise application software has never been an exact science. The study of a desired CRM's features and evaluation criteria is based on a small to medium manufacturing company. A comparison table was prepared for helping the company to select an appropriate vendor. It is noted that a good CRM application must include the following characteristics:

- User Friendly

- Seamless Integration with ERP - to fit the business requirements and the out-ofthe-box functionality for the company

- The best Return On Investment (ROI) Immediate payback with increased productivity, lower sales costs, and streamlined sales operations - all leading to increased revenue. 


\section{References}

[1] Deitel et. al., Web Services A Technical Introduction, 1st Edition, 2003, Prentice Hall, New Jersey.

[2] CRM - A Primer, http://www-1.ibm.com/industries/businesssolutions/crm

[3] Design Guide for CRM Applications Integrating with Microsoft Windows 2000 Active Directory, April, 2001, http://msdn.microsoft.com/library/default.asp?url=/library/enus/dnactdir/html/crm01.asp

[4] CRM Overview, http://www.ittoolbox.com/help/crmovrvierw.asp

[5] Progress, http://www.progress.com/partners/application/our_partners.htm

[6] eBisGen, http://www.bisgen.com/mfgpro.html

[7] Commence, http://www.commence.com

[8] GoldMine, http://www.goldmine.com

[9] Sales Automation, http://www.upshot.com/sales/sales force_automation.html

[10] Marketing Automation, http://www.marketfirst.com/news/press/pivotal.html

http://www.upshot.com/marketing/marketing_automation.html

[11] http://www.salesforce.com/us/products/enterprise-sfa.jsp

[12] The CRM Handbook by Jill Dyche

[13] Customer Relationship Management by Judith W. Kincaid

\section{Biographical}

Melissa Lin is an Information Technology Specialist at the Farm Service Agency of the U.S. Department of Agriculture. Previously, she was an Information Technology Consultant and Coordinator of Computer Applications at the University of Florida. She was in charge of designing and implementing the university's first data warehouse. Before that, she was a Visiting Professor in the Computer Science Department of Indiana University - Purdue University, Fort Wayne, during 1999. And before that, she worked as a Systems Analyst at GTE Data Service, Fort Wayne, Indiana, for 11 years, responsible for E91 1 and CBSS billing supports. Lin's current interests include automated technology, database and Web integration, data warehouse modeling, and Web-based user interface applications. 